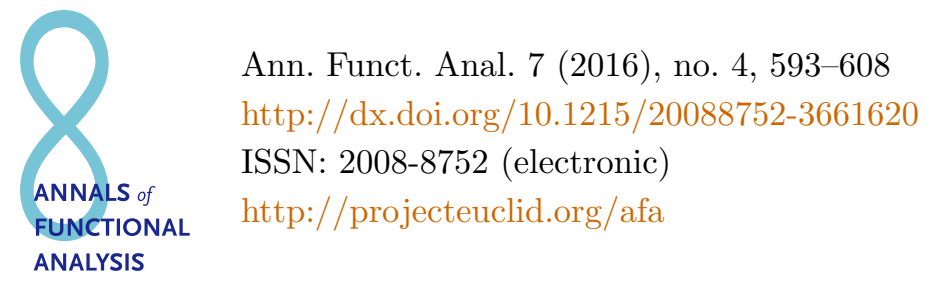

\title{
MAXIMAL BANACH IDEALS OF LIPSCHITZ MAPS
}

\author{
M. G. CABRERA-PADILLA, ${ }^{1}$ J. A. CHÁVEZ-DOMÍNGUEZ, ${ }^{2}$ \\ A. JIMÉNEZ-VARGAS, ${ }^{1 *}$ and MOISÉS VILLEGAS-VALLECILLOS ${ }^{3}$ \\ Communicated by M. Ptak
}

\begin{abstract}
There are known results showing a canonical association between Lipschitz cross-norms (norms on the Lipschitz tensor product of a metric space and a Banach space) and ideals of Lipschitz maps from a metric space to a dual Banach space. We extend this association, relating Lipschitz cross-norms to ideals of Lipschitz maps taking values in general Banach spaces. To do that, we prove a Lipschitz version of the representation theorem for maximal operator ideals. As a consequence, we obtain linear characterizations of some ideals of (nonlinear) Lipschitz maps between metric spaces.
\end{abstract}

\section{INTRODUCTION}

In the theory of Banach spaces, it is well understood that there is a deep connection between norms on tensor products and certain special families of linear maps. The reason is simple: if $E$ and $F$ are vector spaces, a linear functional on $E \otimes F$ can naturally be identified with a linear map from $E$ to the dual of $F$. If $E$ and $F$ are furthermore Banach spaces, a norm on the tensor product $E \otimes F$ will naturally define a collection of bounded linear maps from $E$ to $F^{*}$. With this association in mind, it is possible to develop parallel and interconnected theories between these two worlds: one of norms on tensor products, and one corresponding to these special families of linear maps. Requiring rather minimal conditions for the norm on the tensor product gives rise to collections of linear maps that are in fact ideals - that is, they are closed under composition - and so

Copyright 2016 by the Tusi Mathematical Research Group.

Received Jan. 29, 2016; Accepted Apr. 16, 2016.

${ }^{*}$ Corresponding author.

2010 Mathematics Subject Classification. Primary 46B28; Secondary 26A16, 46E15, 47L20.

Keywords. Lipschitz map, tensor product, $p$-summing operator, duality, ideal. 


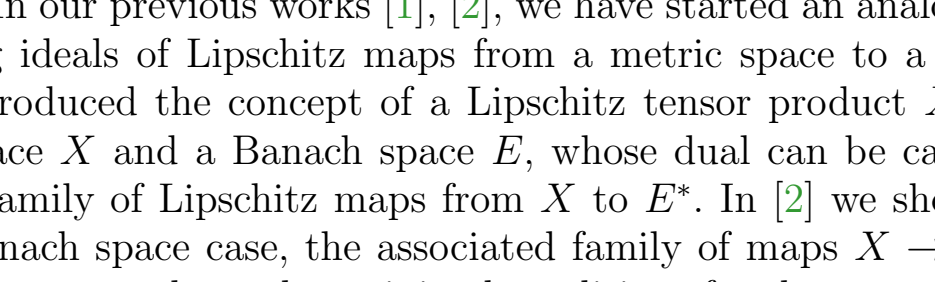

\title{
MAXIMAL BANACH IDEALS OF LIPSCHITZ MAPS
}

\author{
M. G. CABRERA-PADILLA, ${ }^{1}$ J. A. CHÁVEZ-DOMÍNGUEZ, ${ }^{2}$ \\ A. JIMÉNEZ-VARGAS, ${ }^{1 *}$ and MOISÉS VILLEGAS-VALLECILLOS ${ }^{3}$ \\ Communicated by M. Ptak
}

\begin{abstract}
There are known results showing a canonical association between Lipschitz cross-norms (norms on the Lipschitz tensor product of a metric space and a Banach space) and ideals of Lipschitz maps from a metric space to a dual Banach space. We extend this association, relating Lipschitz cross-norms to ideals of Lipschitz maps taking values in general Banach spaces. To do that, we prove a Lipschitz version of the representation theorem for maximal operator ideals. As a consequence, we obtain linear characterizations of some ideals of (nonlinear) Lipschitz maps between metric spaces.
\end{abstract}

\section{INTRODUCTION}

In the theory of Banach spaces, it is well understood that there is a deep connection between norms on tensor products and certain special families of linear maps. The reason is simple: if $E$ and $F$ are vector spaces, a linear functional on $E \otimes F$ can naturally be identified with a linear map from $E$ to the dual of $F$. If $E$ and $F$ are furthermore Banach spaces, a norm on the tensor product $E \otimes F$ will naturally define a collection of bounded linear maps from $E$ to $F^{*}$. With this association in mind, it is possible to develop parallel and interconnected theories between these two worlds: one of norms on tensor products, and one corresponding to these special families of linear maps. Requiring rather minimal conditions for the norm on the tensor product gives rise to collections of linear maps that are in fact ideals - that is, they are closed under composition - and so

Copyright 2016 by the Tusi Mathematical Research Group.

Received Jan. 29, 2016; Accepted Apr. 16, 2016.

${ }^{*}$ Corresponding author.

2010 Mathematics Subject Classification. Primary 46B28; Secondary 26A16, 46E15, 47L20.

Keywords. Lipschitz map, tensor product, $p$-summing operator, duality, ideal. 


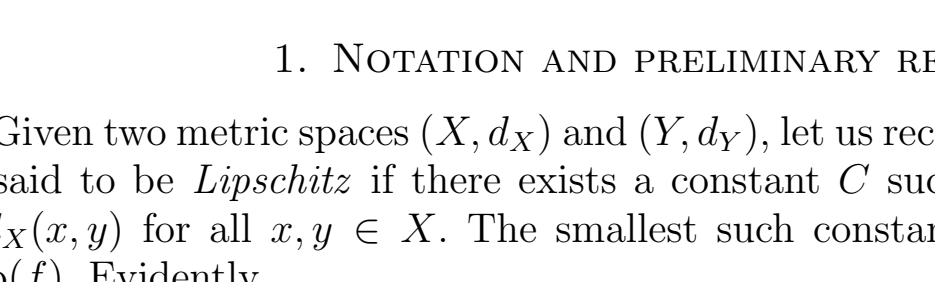

\title{
MAXIMAL BANACH IDEALS OF LIPSCHITZ MAPS
}

\author{
M. G. CABRERA-PADILLA, ${ }^{1}$ J. A. CHÁVEZ-DOMÍNGUEZ, ${ }^{2}$ \\ A. JIMÉNEZ-VARGAS, ${ }^{1 *}$ and MOISÉS VILLEGAS-VALLECILLOS ${ }^{3}$ \\ Communicated by M. Ptak
}

\begin{abstract}
There are known results showing a canonical association between Lipschitz cross-norms (norms on the Lipschitz tensor product of a metric space and a Banach space) and ideals of Lipschitz maps from a metric space to a dual Banach space. We extend this association, relating Lipschitz cross-norms to ideals of Lipschitz maps taking values in general Banach spaces. To do that, we prove a Lipschitz version of the representation theorem for maximal operator ideals. As a consequence, we obtain linear characterizations of some ideals of (nonlinear) Lipschitz maps between metric spaces.
\end{abstract}

\section{INTRODUCTION}

In the theory of Banach spaces, it is well understood that there is a deep connection between norms on tensor products and certain special families of linear maps. The reason is simple: if $E$ and $F$ are vector spaces, a linear functional on $E \otimes F$ can naturally be identified with a linear map from $E$ to the dual of $F$. If $E$ and $F$ are furthermore Banach spaces, a norm on the tensor product $E \otimes F$ will naturally define a collection of bounded linear maps from $E$ to $F^{*}$. With this association in mind, it is possible to develop parallel and interconnected theories between these two worlds: one of norms on tensor products, and one corresponding to these special families of linear maps. Requiring rather minimal conditions for the norm on the tensor product gives rise to collections of linear maps that are in fact ideals - that is, they are closed under composition - and so

Copyright 2016 by the Tusi Mathematical Research Group.

Received Jan. 29, 2016; Accepted Apr. 16, 2016.

${ }^{*}$ Corresponding author.

2010 Mathematics Subject Classification. Primary 46B28; Secondary 26A16, 46E15, 47L20.

Keywords. Lipschitz map, tensor product, $p$-summing operator, duality, ideal. 


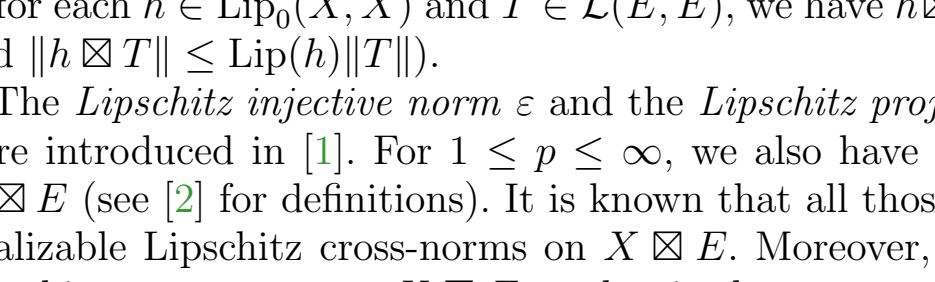

\title{
MAXIMAL BANACH IDEALS OF LIPSCHITZ MAPS
}

\author{
M. G. CABRERA-PADILLA, ${ }^{1}$ J. A. CHÁVEZ-DOMÍNGUEZ, ${ }^{2}$ \\ A. JIMÉNEZ-VARGAS, ${ }^{1 *}$ and MOISÉS VILLEGAS-VALLECILLOS ${ }^{3}$ \\ Communicated by M. Ptak
}

\begin{abstract}
There are known results showing a canonical association between Lipschitz cross-norms (norms on the Lipschitz tensor product of a metric space and a Banach space) and ideals of Lipschitz maps from a metric space to a dual Banach space. We extend this association, relating Lipschitz cross-norms to ideals of Lipschitz maps taking values in general Banach spaces. To do that, we prove a Lipschitz version of the representation theorem for maximal operator ideals. As a consequence, we obtain linear characterizations of some ideals of (nonlinear) Lipschitz maps between metric spaces.
\end{abstract}

\section{INTRODUCTION}

In the theory of Banach spaces, it is well understood that there is a deep connection between norms on tensor products and certain special families of linear maps. The reason is simple: if $E$ and $F$ are vector spaces, a linear functional on $E \otimes F$ can naturally be identified with a linear map from $E$ to the dual of $F$. If $E$ and $F$ are furthermore Banach spaces, a norm on the tensor product $E \otimes F$ will naturally define a collection of bounded linear maps from $E$ to $F^{*}$. With this association in mind, it is possible to develop parallel and interconnected theories between these two worlds: one of norms on tensor products, and one corresponding to these special families of linear maps. Requiring rather minimal conditions for the norm on the tensor product gives rise to collections of linear maps that are in fact ideals - that is, they are closed under composition - and so

Copyright 2016 by the Tusi Mathematical Research Group.

Received Jan. 29, 2016; Accepted Apr. 16, 2016.

${ }^{*}$ Corresponding author.

2010 Mathematics Subject Classification. Primary 46B28; Secondary 26A16, 46E15, 47L20.

Keywords. Lipschitz map, tensor product, $p$-summing operator, duality, ideal. 


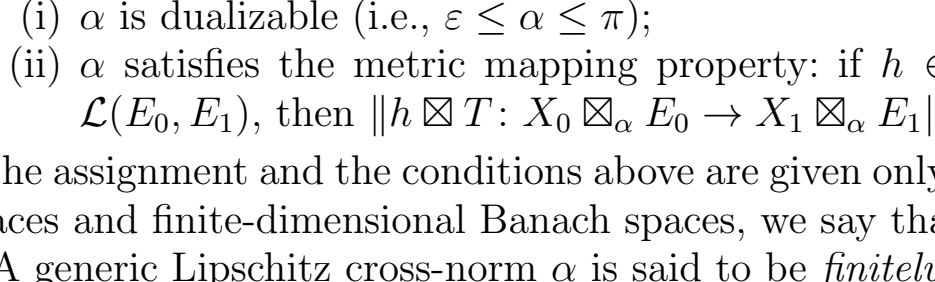

\title{
MAXIMAL BANACH IDEALS OF LIPSCHITZ MAPS
}

\author{
M. G. CABRERA-PADILLA, ${ }^{1}$ J. A. CHÁVEZ-DOMÍNGUEZ, ${ }^{2}$ \\ A. JIMÉNEZ-VARGAS, ${ }^{1 *}$ and MOISÉS VILLEGAS-VALLECILLOS ${ }^{3}$ \\ Communicated by M. Ptak
}

\begin{abstract}
There are known results showing a canonical association between Lipschitz cross-norms (norms on the Lipschitz tensor product of a metric space and a Banach space) and ideals of Lipschitz maps from a metric space to a dual Banach space. We extend this association, relating Lipschitz cross-norms to ideals of Lipschitz maps taking values in general Banach spaces. To do that, we prove a Lipschitz version of the representation theorem for maximal operator ideals. As a consequence, we obtain linear characterizations of some ideals of (nonlinear) Lipschitz maps between metric spaces.
\end{abstract}

\section{INTRODUCTION}

In the theory of Banach spaces, it is well understood that there is a deep connection between norms on tensor products and certain special families of linear maps. The reason is simple: if $E$ and $F$ are vector spaces, a linear functional on $E \otimes F$ can naturally be identified with a linear map from $E$ to the dual of $F$. If $E$ and $F$ are furthermore Banach spaces, a norm on the tensor product $E \otimes F$ will naturally define a collection of bounded linear maps from $E$ to $F^{*}$. With this association in mind, it is possible to develop parallel and interconnected theories between these two worlds: one of norms on tensor products, and one corresponding to these special families of linear maps. Requiring rather minimal conditions for the norm on the tensor product gives rise to collections of linear maps that are in fact ideals - that is, they are closed under composition - and so

Copyright 2016 by the Tusi Mathematical Research Group.

Received Jan. 29, 2016; Accepted Apr. 16, 2016.

${ }^{*}$ Corresponding author.

2010 Mathematics Subject Classification. Primary 46B28; Secondary 26A16, 46E15, 47L20.

Keywords. Lipschitz map, tensor product, $p$-summing operator, duality, ideal. 


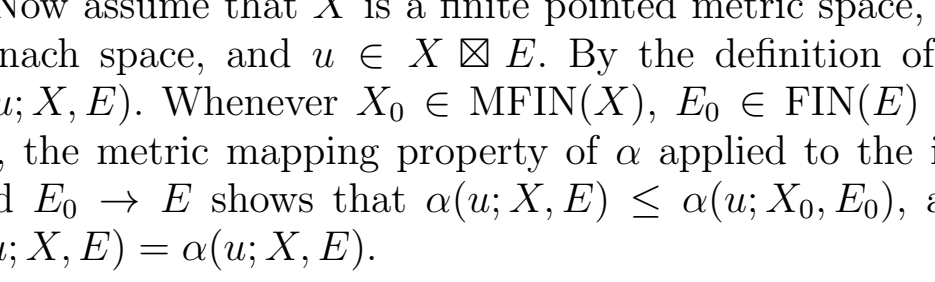

\title{
MAXIMAL BANACH IDEALS OF LIPSCHITZ MAPS
}

\author{
M. G. CABRERA-PADILLA, ${ }^{1}$ J. A. CHÁVEZ-DOMÍNGUEZ, ${ }^{2}$ \\ A. JIMÉNEZ-VARGAS, ${ }^{1 *}$ and MOISÉS VILLEGAS-VALLECILLOS ${ }^{3}$ \\ Communicated by M. Ptak
}

\begin{abstract}
There are known results showing a canonical association between Lipschitz cross-norms (norms on the Lipschitz tensor product of a metric space and a Banach space) and ideals of Lipschitz maps from a metric space to a dual Banach space. We extend this association, relating Lipschitz cross-norms to ideals of Lipschitz maps taking values in general Banach spaces. To do that, we prove a Lipschitz version of the representation theorem for maximal operator ideals. As a consequence, we obtain linear characterizations of some ideals of (nonlinear) Lipschitz maps between metric spaces.
\end{abstract}

\section{INTRODUCTION}

In the theory of Banach spaces, it is well understood that there is a deep connection between norms on tensor products and certain special families of linear maps. The reason is simple: if $E$ and $F$ are vector spaces, a linear functional on $E \otimes F$ can naturally be identified with a linear map from $E$ to the dual of $F$. If $E$ and $F$ are furthermore Banach spaces, a norm on the tensor product $E \otimes F$ will naturally define a collection of bounded linear maps from $E$ to $F^{*}$. With this association in mind, it is possible to develop parallel and interconnected theories between these two worlds: one of norms on tensor products, and one corresponding to these special families of linear maps. Requiring rather minimal conditions for the norm on the tensor product gives rise to collections of linear maps that are in fact ideals - that is, they are closed under composition - and so

Copyright 2016 by the Tusi Mathematical Research Group.

Received Jan. 29, 2016; Accepted Apr. 16, 2016.

${ }^{*}$ Corresponding author.

2010 Mathematics Subject Classification. Primary 46B28; Secondary 26A16, 46E15, 47L20.

Keywords. Lipschitz map, tensor product, $p$-summing operator, duality, ideal. 


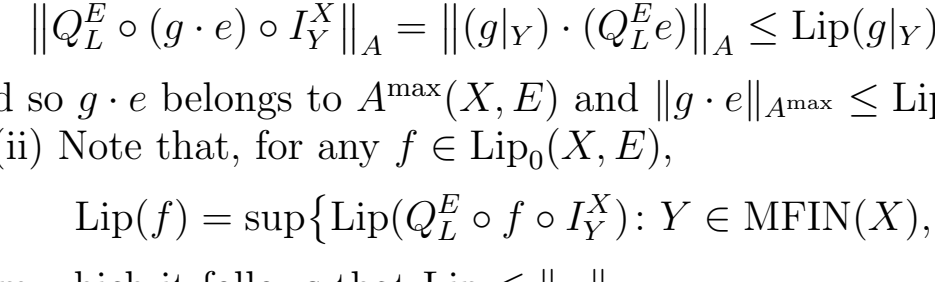

\title{
MAXIMAL BANACH IDEALS OF LIPSCHITZ MAPS
}

\author{
M. G. CABRERA-PADILLA, ${ }^{1}$ J. A. CHÁVEZ-DOMÍNGUEZ, ${ }^{2}$ \\ A. JIMÉNEZ-VARGAS, ${ }^{1 *}$ and MOISÉS VILLEGAS-VALLECILLOS ${ }^{3}$ \\ Communicated by M. Ptak
}

\begin{abstract}
There are known results showing a canonical association between Lipschitz cross-norms (norms on the Lipschitz tensor product of a metric space and a Banach space) and ideals of Lipschitz maps from a metric space to a dual Banach space. We extend this association, relating Lipschitz cross-norms to ideals of Lipschitz maps taking values in general Banach spaces. To do that, we prove a Lipschitz version of the representation theorem for maximal operator ideals. As a consequence, we obtain linear characterizations of some ideals of (nonlinear) Lipschitz maps between metric spaces.
\end{abstract}

\section{INTRODUCTION}

In the theory of Banach spaces, it is well understood that there is a deep connection between norms on tensor products and certain special families of linear maps. The reason is simple: if $E$ and $F$ are vector spaces, a linear functional on $E \otimes F$ can naturally be identified with a linear map from $E$ to the dual of $F$. If $E$ and $F$ are furthermore Banach spaces, a norm on the tensor product $E \otimes F$ will naturally define a collection of bounded linear maps from $E$ to $F^{*}$. With this association in mind, it is possible to develop parallel and interconnected theories between these two worlds: one of norms on tensor products, and one corresponding to these special families of linear maps. Requiring rather minimal conditions for the norm on the tensor product gives rise to collections of linear maps that are in fact ideals - that is, they are closed under composition - and so

Copyright 2016 by the Tusi Mathematical Research Group.

Received Jan. 29, 2016; Accepted Apr. 16, 2016.

${ }^{*}$ Corresponding author.

2010 Mathematics Subject Classification. Primary 46B28; Secondary 26A16, 46E15, 47L20.

Keywords. Lipschitz map, tensor product, $p$-summing operator, duality, ideal. 


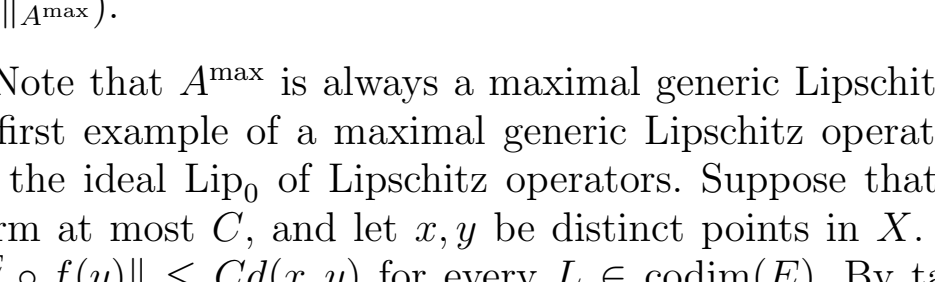

\title{
MAXIMAL BANACH IDEALS OF LIPSCHITZ MAPS
}

\author{
M. G. CABRERA-PADILLA, ${ }^{1}$ J. A. CHÁVEZ-DOMÍNGUEZ, ${ }^{2}$ \\ A. JIMÉNEZ-VARGAS, ${ }^{1 *}$ and MOISÉS VILLEGAS-VALLECILLOS ${ }^{3}$ \\ Communicated by M. Ptak
}

\begin{abstract}
There are known results showing a canonical association between Lipschitz cross-norms (norms on the Lipschitz tensor product of a metric space and a Banach space) and ideals of Lipschitz maps from a metric space to a dual Banach space. We extend this association, relating Lipschitz cross-norms to ideals of Lipschitz maps taking values in general Banach spaces. To do that, we prove a Lipschitz version of the representation theorem for maximal operator ideals. As a consequence, we obtain linear characterizations of some ideals of (nonlinear) Lipschitz maps between metric spaces.
\end{abstract}

\section{INTRODUCTION}

In the theory of Banach spaces, it is well understood that there is a deep connection between norms on tensor products and certain special families of linear maps. The reason is simple: if $E$ and $F$ are vector spaces, a linear functional on $E \otimes F$ can naturally be identified with a linear map from $E$ to the dual of $F$. If $E$ and $F$ are furthermore Banach spaces, a norm on the tensor product $E \otimes F$ will naturally define a collection of bounded linear maps from $E$ to $F^{*}$. With this association in mind, it is possible to develop parallel and interconnected theories between these two worlds: one of norms on tensor products, and one corresponding to these special families of linear maps. Requiring rather minimal conditions for the norm on the tensor product gives rise to collections of linear maps that are in fact ideals - that is, they are closed under composition - and so

Copyright 2016 by the Tusi Mathematical Research Group.

Received Jan. 29, 2016; Accepted Apr. 16, 2016.

${ }^{*}$ Corresponding author.

2010 Mathematics Subject Classification. Primary 46B28; Secondary 26A16, 46E15, 47L20.

Keywords. Lipschitz map, tensor product, $p$-summing operator, duality, ideal. 


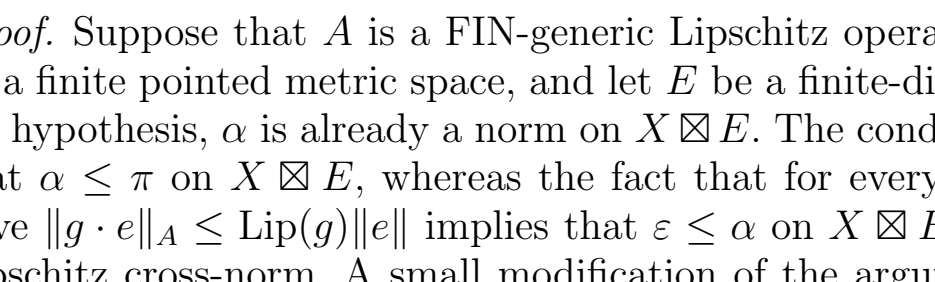

\title{
MAXIMAL BANACH IDEALS OF LIPSCHITZ MAPS
}

\author{
M. G. CABRERA-PADILLA, ${ }^{1}$ J. A. CHÁVEZ-DOMÍNGUEZ, ${ }^{2}$ \\ A. JIMÉNEZ-VARGAS, ${ }^{1 *}$ and MOISÉS VILLEGAS-VALLECILLOS ${ }^{3}$ \\ Communicated by M. Ptak
}

\begin{abstract}
There are known results showing a canonical association between Lipschitz cross-norms (norms on the Lipschitz tensor product of a metric space and a Banach space) and ideals of Lipschitz maps from a metric space to a dual Banach space. We extend this association, relating Lipschitz cross-norms to ideals of Lipschitz maps taking values in general Banach spaces. To do that, we prove a Lipschitz version of the representation theorem for maximal operator ideals. As a consequence, we obtain linear characterizations of some ideals of (nonlinear) Lipschitz maps between metric spaces.
\end{abstract}

\section{INTRODUCTION}

In the theory of Banach spaces, it is well understood that there is a deep connection between norms on tensor products and certain special families of linear maps. The reason is simple: if $E$ and $F$ are vector spaces, a linear functional on $E \otimes F$ can naturally be identified with a linear map from $E$ to the dual of $F$. If $E$ and $F$ are furthermore Banach spaces, a norm on the tensor product $E \otimes F$ will naturally define a collection of bounded linear maps from $E$ to $F^{*}$. With this association in mind, it is possible to develop parallel and interconnected theories between these two worlds: one of norms on tensor products, and one corresponding to these special families of linear maps. Requiring rather minimal conditions for the norm on the tensor product gives rise to collections of linear maps that are in fact ideals - that is, they are closed under composition - and so

Copyright 2016 by the Tusi Mathematical Research Group.

Received Jan. 29, 2016; Accepted Apr. 16, 2016.

${ }^{*}$ Corresponding author.

2010 Mathematics Subject Classification. Primary 46B28; Secondary 26A16, 46E15, 47L20.

Keywords. Lipschitz map, tensor product, $p$-summing operator, duality, ideal. 


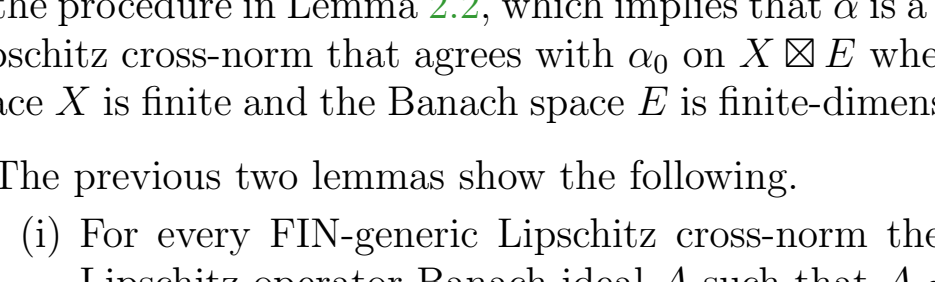

\title{
MAXIMAL BANACH IDEALS OF LIPSCHITZ MAPS
}

\author{
M. G. CABRERA-PADILLA, ${ }^{1}$ J. A. CHÁVEZ-DOMÍNGUEZ, ${ }^{2}$ \\ A. JIMÉNEZ-VARGAS, ${ }^{1 *}$ and MOISÉS VILLEGAS-VALLECILLOS ${ }^{3}$ \\ Communicated by M. Ptak
}

\begin{abstract}
There are known results showing a canonical association between Lipschitz cross-norms (norms on the Lipschitz tensor product of a metric space and a Banach space) and ideals of Lipschitz maps from a metric space to a dual Banach space. We extend this association, relating Lipschitz cross-norms to ideals of Lipschitz maps taking values in general Banach spaces. To do that, we prove a Lipschitz version of the representation theorem for maximal operator ideals. As a consequence, we obtain linear characterizations of some ideals of (nonlinear) Lipschitz maps between metric spaces.
\end{abstract}

\section{INTRODUCTION}

In the theory of Banach spaces, it is well understood that there is a deep connection between norms on tensor products and certain special families of linear maps. The reason is simple: if $E$ and $F$ are vector spaces, a linear functional on $E \otimes F$ can naturally be identified with a linear map from $E$ to the dual of $F$. If $E$ and $F$ are furthermore Banach spaces, a norm on the tensor product $E \otimes F$ will naturally define a collection of bounded linear maps from $E$ to $F^{*}$. With this association in mind, it is possible to develop parallel and interconnected theories between these two worlds: one of norms on tensor products, and one corresponding to these special families of linear maps. Requiring rather minimal conditions for the norm on the tensor product gives rise to collections of linear maps that are in fact ideals - that is, they are closed under composition - and so

Copyright 2016 by the Tusi Mathematical Research Group.

Received Jan. 29, 2016; Accepted Apr. 16, 2016.

${ }^{*}$ Corresponding author.

2010 Mathematics Subject Classification. Primary 46B28; Secondary 26A16, 46E15, 47L20.

Keywords. Lipschitz map, tensor product, $p$-summing operator, duality, ideal. 


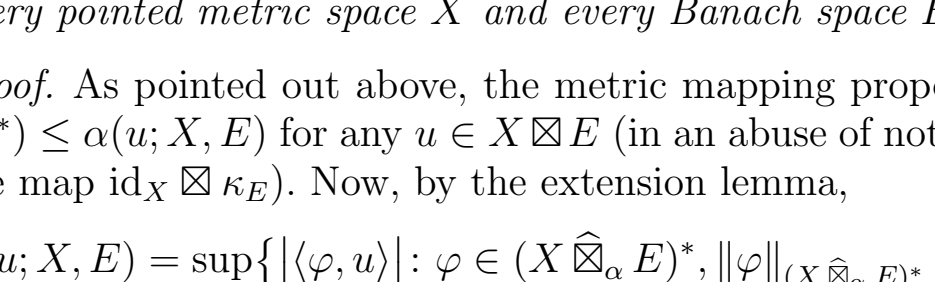

\title{
MAXIMAL BANACH IDEALS OF LIPSCHITZ MAPS
}

\author{
M. G. CABRERA-PADILLA, ${ }^{1}$ J. A. CHÁVEZ-DOMÍNGUEZ, ${ }^{2}$ \\ A. JIMÉNEZ-VARGAS, ${ }^{1 *}$ and MOISÉS VILLEGAS-VALLECILLOS ${ }^{3}$ \\ Communicated by M. Ptak
}

\begin{abstract}
There are known results showing a canonical association between Lipschitz cross-norms (norms on the Lipschitz tensor product of a metric space and a Banach space) and ideals of Lipschitz maps from a metric space to a dual Banach space. We extend this association, relating Lipschitz cross-norms to ideals of Lipschitz maps taking values in general Banach spaces. To do that, we prove a Lipschitz version of the representation theorem for maximal operator ideals. As a consequence, we obtain linear characterizations of some ideals of (nonlinear) Lipschitz maps between metric spaces.
\end{abstract}

\section{INTRODUCTION}

In the theory of Banach spaces, it is well understood that there is a deep connection between norms on tensor products and certain special families of linear maps. The reason is simple: if $E$ and $F$ are vector spaces, a linear functional on $E \otimes F$ can naturally be identified with a linear map from $E$ to the dual of $F$. If $E$ and $F$ are furthermore Banach spaces, a norm on the tensor product $E \otimes F$ will naturally define a collection of bounded linear maps from $E$ to $F^{*}$. With this association in mind, it is possible to develop parallel and interconnected theories between these two worlds: one of norms on tensor products, and one corresponding to these special families of linear maps. Requiring rather minimal conditions for the norm on the tensor product gives rise to collections of linear maps that are in fact ideals - that is, they are closed under composition - and so

Copyright 2016 by the Tusi Mathematical Research Group.

Received Jan. 29, 2016; Accepted Apr. 16, 2016.

${ }^{*}$ Corresponding author.

2010 Mathematics Subject Classification. Primary 46B28; Secondary 26A16, 46E15, 47L20.

Keywords. Lipschitz map, tensor product, $p$-summing operator, duality, ideal. 


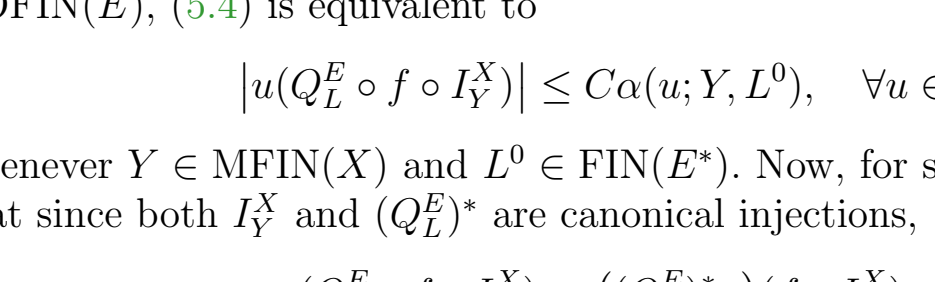

\title{
MAXIMAL BANACH IDEALS OF LIPSCHITZ MAPS
}

\author{
M. G. CABRERA-PADILLA, ${ }^{1}$ J. A. CHÁVEZ-DOMÍNGUEZ, ${ }^{2}$ \\ A. JIMÉNEZ-VARGAS, ${ }^{1 *}$ and MOISÉS VILLEGAS-VALLECILLOS ${ }^{3}$ \\ Communicated by M. Ptak
}

\begin{abstract}
There are known results showing a canonical association between Lipschitz cross-norms (norms on the Lipschitz tensor product of a metric space and a Banach space) and ideals of Lipschitz maps from a metric space to a dual Banach space. We extend this association, relating Lipschitz cross-norms to ideals of Lipschitz maps taking values in general Banach spaces. To do that, we prove a Lipschitz version of the representation theorem for maximal operator ideals. As a consequence, we obtain linear characterizations of some ideals of (nonlinear) Lipschitz maps between metric spaces.
\end{abstract}

\section{INTRODUCTION}

In the theory of Banach spaces, it is well understood that there is a deep connection between norms on tensor products and certain special families of linear maps. The reason is simple: if $E$ and $F$ are vector spaces, a linear functional on $E \otimes F$ can naturally be identified with a linear map from $E$ to the dual of $F$. If $E$ and $F$ are furthermore Banach spaces, a norm on the tensor product $E \otimes F$ will naturally define a collection of bounded linear maps from $E$ to $F^{*}$. With this association in mind, it is possible to develop parallel and interconnected theories between these two worlds: one of norms on tensor products, and one corresponding to these special families of linear maps. Requiring rather minimal conditions for the norm on the tensor product gives rise to collections of linear maps that are in fact ideals - that is, they are closed under composition - and so

Copyright 2016 by the Tusi Mathematical Research Group.

Received Jan. 29, 2016; Accepted Apr. 16, 2016.

${ }^{*}$ Corresponding author.

2010 Mathematics Subject Classification. Primary 46B28; Secondary 26A16, 46E15, 47L20.

Keywords. Lipschitz map, tensor product, $p$-summing operator, duality, ideal. 


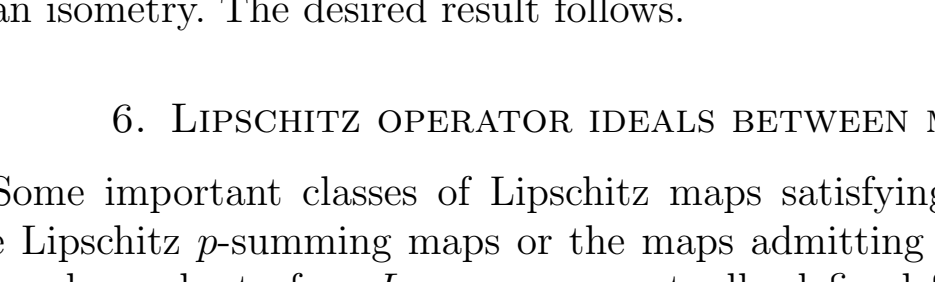

\title{
MAXIMAL BANACH IDEALS OF LIPSCHITZ MAPS
}

\author{
M. G. CABRERA-PADILLA, ${ }^{1}$ J. A. CHÁVEZ-DOMÍNGUEZ, ${ }^{2}$ \\ A. JIMÉNEZ-VARGAS, ${ }^{1 *}$ and MOISÉS VILLEGAS-VALLECILLOS ${ }^{3}$ \\ Communicated by M. Ptak
}

\begin{abstract}
There are known results showing a canonical association between Lipschitz cross-norms (norms on the Lipschitz tensor product of a metric space and a Banach space) and ideals of Lipschitz maps from a metric space to a dual Banach space. We extend this association, relating Lipschitz cross-norms to ideals of Lipschitz maps taking values in general Banach spaces. To do that, we prove a Lipschitz version of the representation theorem for maximal operator ideals. As a consequence, we obtain linear characterizations of some ideals of (nonlinear) Lipschitz maps between metric spaces.
\end{abstract}

\section{INTRODUCTION}

In the theory of Banach spaces, it is well understood that there is a deep connection between norms on tensor products and certain special families of linear maps. The reason is simple: if $E$ and $F$ are vector spaces, a linear functional on $E \otimes F$ can naturally be identified with a linear map from $E$ to the dual of $F$. If $E$ and $F$ are furthermore Banach spaces, a norm on the tensor product $E \otimes F$ will naturally define a collection of bounded linear maps from $E$ to $F^{*}$. With this association in mind, it is possible to develop parallel and interconnected theories between these two worlds: one of norms on tensor products, and one corresponding to these special families of linear maps. Requiring rather minimal conditions for the norm on the tensor product gives rise to collections of linear maps that are in fact ideals - that is, they are closed under composition - and so

Copyright 2016 by the Tusi Mathematical Research Group.

Received Jan. 29, 2016; Accepted Apr. 16, 2016.

${ }^{*}$ Corresponding author.

2010 Mathematics Subject Classification. Primary 46B28; Secondary 26A16, 46E15, 47L20.

Keywords. Lipschitz map, tensor product, $p$-summing operator, duality, ideal. 


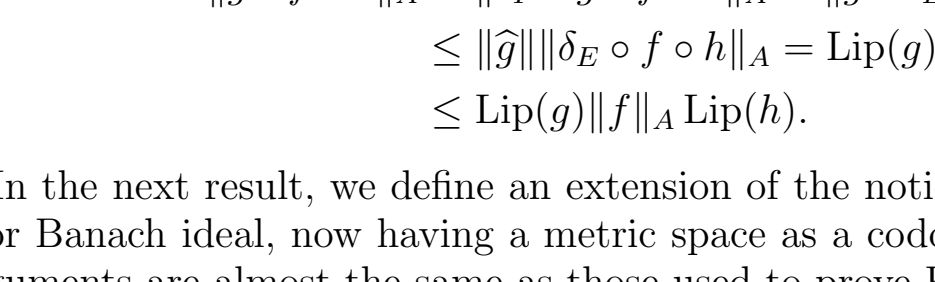

\title{
MAXIMAL BANACH IDEALS OF LIPSCHITZ MAPS
}

\author{
M. G. CABRERA-PADILLA, ${ }^{1}$ J. A. CHÁVEZ-DOMÍNGUEZ, ${ }^{2}$ \\ A. JIMÉNEZ-VARGAS, ${ }^{1 *}$ and MOISÉS VILLEGAS-VALLECILLOS ${ }^{3}$ \\ Communicated by M. Ptak
}

\begin{abstract}
There are known results showing a canonical association between Lipschitz cross-norms (norms on the Lipschitz tensor product of a metric space and a Banach space) and ideals of Lipschitz maps from a metric space to a dual Banach space. We extend this association, relating Lipschitz cross-norms to ideals of Lipschitz maps taking values in general Banach spaces. To do that, we prove a Lipschitz version of the representation theorem for maximal operator ideals. As a consequence, we obtain linear characterizations of some ideals of (nonlinear) Lipschitz maps between metric spaces.
\end{abstract}

\section{INTRODUCTION}

In the theory of Banach spaces, it is well understood that there is a deep connection between norms on tensor products and certain special families of linear maps. The reason is simple: if $E$ and $F$ are vector spaces, a linear functional on $E \otimes F$ can naturally be identified with a linear map from $E$ to the dual of $F$. If $E$ and $F$ are furthermore Banach spaces, a norm on the tensor product $E \otimes F$ will naturally define a collection of bounded linear maps from $E$ to $F^{*}$. With this association in mind, it is possible to develop parallel and interconnected theories between these two worlds: one of norms on tensor products, and one corresponding to these special families of linear maps. Requiring rather minimal conditions for the norm on the tensor product gives rise to collections of linear maps that are in fact ideals - that is, they are closed under composition - and so

Copyright 2016 by the Tusi Mathematical Research Group.

Received Jan. 29, 2016; Accepted Apr. 16, 2016.

${ }^{*}$ Corresponding author.

2010 Mathematics Subject Classification. Primary 46B28; Secondary 26A16, 46E15, 47L20.

Keywords. Lipschitz map, tensor product, $p$-summing operator, duality, ideal. 


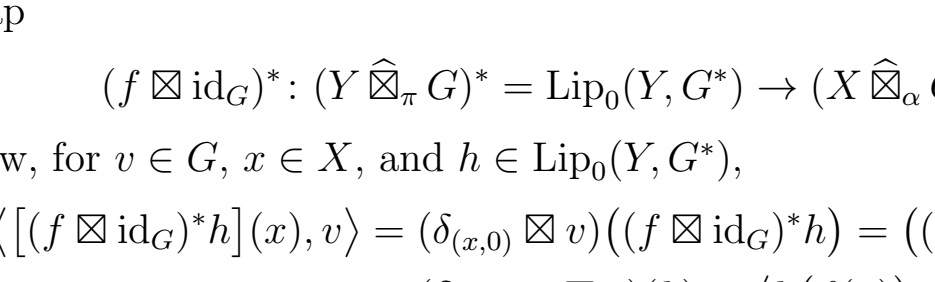

\title{
MAXIMAL BANACH IDEALS OF LIPSCHITZ MAPS
}

\author{
M. G. CABRERA-PADILLA, ${ }^{1}$ J. A. CHÁVEZ-DOMÍNGUEZ, ${ }^{2}$ \\ A. JIMÉNEZ-VARGAS, ${ }^{1 *}$ and MOISÉS VILLEGAS-VALLECILLOS ${ }^{3}$ \\ Communicated by M. Ptak
}

\begin{abstract}
There are known results showing a canonical association between Lipschitz cross-norms (norms on the Lipschitz tensor product of a metric space and a Banach space) and ideals of Lipschitz maps from a metric space to a dual Banach space. We extend this association, relating Lipschitz cross-norms to ideals of Lipschitz maps taking values in general Banach spaces. To do that, we prove a Lipschitz version of the representation theorem for maximal operator ideals. As a consequence, we obtain linear characterizations of some ideals of (nonlinear) Lipschitz maps between metric spaces.
\end{abstract}

\section{INTRODUCTION}

In the theory of Banach spaces, it is well understood that there is a deep connection between norms on tensor products and certain special families of linear maps. The reason is simple: if $E$ and $F$ are vector spaces, a linear functional on $E \otimes F$ can naturally be identified with a linear map from $E$ to the dual of $F$. If $E$ and $F$ are furthermore Banach spaces, a norm on the tensor product $E \otimes F$ will naturally define a collection of bounded linear maps from $E$ to $F^{*}$. With this association in mind, it is possible to develop parallel and interconnected theories between these two worlds: one of norms on tensor products, and one corresponding to these special families of linear maps. Requiring rather minimal conditions for the norm on the tensor product gives rise to collections of linear maps that are in fact ideals - that is, they are closed under composition - and so

Copyright 2016 by the Tusi Mathematical Research Group.

Received Jan. 29, 2016; Accepted Apr. 16, 2016.

${ }^{*}$ Corresponding author.

2010 Mathematics Subject Classification. Primary 46B28; Secondary 26A16, 46E15, 47L20.

Keywords. Lipschitz map, tensor product, $p$-summing operator, duality, ideal. 


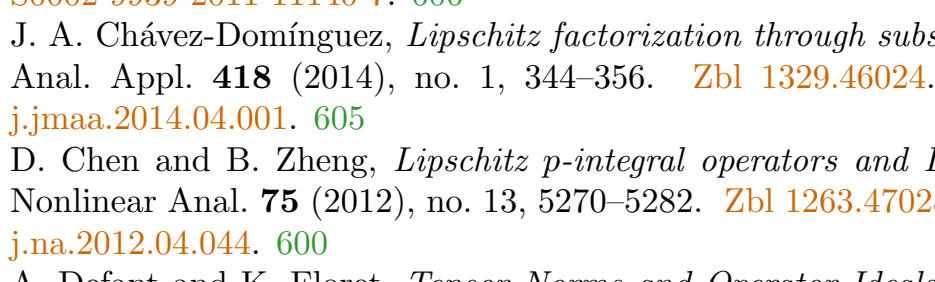

\title{
MAXIMAL BANACH IDEALS OF LIPSCHITZ MAPS
}

\author{
M. G. CABRERA-PADILLA, ${ }^{1}$ J. A. CHÁVEZ-DOMÍNGUEZ, ${ }^{2}$ \\ A. JIMÉNEZ-VARGAS, ${ }^{1 *}$ and MOISÉS VILLEGAS-VALLECILLOS ${ }^{3}$ \\ Communicated by M. Ptak
}

\begin{abstract}
There are known results showing a canonical association between Lipschitz cross-norms (norms on the Lipschitz tensor product of a metric space and a Banach space) and ideals of Lipschitz maps from a metric space to a dual Banach space. We extend this association, relating Lipschitz cross-norms to ideals of Lipschitz maps taking values in general Banach spaces. To do that, we prove a Lipschitz version of the representation theorem for maximal operator ideals. As a consequence, we obtain linear characterizations of some ideals of (nonlinear) Lipschitz maps between metric spaces.
\end{abstract}

\section{INTRODUCTION}

In the theory of Banach spaces, it is well understood that there is a deep connection between norms on tensor products and certain special families of linear maps. The reason is simple: if $E$ and $F$ are vector spaces, a linear functional on $E \otimes F$ can naturally be identified with a linear map from $E$ to the dual of $F$. If $E$ and $F$ are furthermore Banach spaces, a norm on the tensor product $E \otimes F$ will naturally define a collection of bounded linear maps from $E$ to $F^{*}$. With this association in mind, it is possible to develop parallel and interconnected theories between these two worlds: one of norms on tensor products, and one corresponding to these special families of linear maps. Requiring rather minimal conditions for the norm on the tensor product gives rise to collections of linear maps that are in fact ideals - that is, they are closed under composition - and so

Copyright 2016 by the Tusi Mathematical Research Group.

Received Jan. 29, 2016; Accepted Apr. 16, 2016.

${ }^{*}$ Corresponding author.

2010 Mathematics Subject Classification. Primary 46B28; Secondary 26A16, 46E15, 47L20.

Keywords. Lipschitz map, tensor product, $p$-summing operator, duality, ideal. 